\title{
A generalized spherical version of the Blume-Emery-Griffiths model with ferromagnetic and antiferromagnetic interactions.
}

\author{
S.E. Savel'ev ${ }^{a}$ and Guillermo Ramírez-Santiago ${ }^{b}$ \\ (a) All-Russian Electrical Engineering Institute, Moscow, Russia, and Institute of Materials \\ Science, University of Tsukuba, 1-1-1 Tennodai, Tsukuba 305-8573, Japan, \\ (b) Instituto de Fisica, Universidad Nacional Autónoma de México, PO Box 20-364 México, \\ 01000, D.F. MEXICO
}

\begin{abstract}
We have investigated analitycally the phase diagram of a generalized spherical version of the Blume-Emery-Griffiths model that includes ferromagnetic or antiferromagnetic spin interactions as well as quadrupole interactions in zero and nonzero magnetic field. We show that in three dimensions and zero magnetic field a regular paramagnetic-ferromagnetic (PM-FM) or a paramagnetic-antiferromagnetic (PM-AFM) phase transition occurs whenever the magnetic spin interactions dominate over the quadrupole interactions. However, when spin and quadrupole interactions are important, there appears a reentrant FM-PM or AFM-PM phase transition at low temperatures, in addition to the regular PM-FM or PM-AFM phase transitions. On the other hand, in a nonzero homogeneous external magnetic field $H$, we find no evidence of a transition to the state with spontaneous magnetization for FM interactions in three dimensions. Nonethelesss, for AFM interactions we do get a scenario similar to that described above for zero external magnetic field, except that the critical temperatures are now functions of $H$. We also find two critical field values, $H_{c 1}$, at which the reentrance phenomenon dissapears and $H_{c 2}\left(H_{c 1} \approx 0.5 H_{c 2}\right)$, above which the PM-AFM transition temperature vanishes.
\end{abstract}

PACS 75.10.Hk, 05.20.-y, 05.50.+q, 05.70.Fh, 05.70.Jk 


\section{INTRODUCTION}

Continuous spins models play an important role as approximate statistical mechanical models for several physical systems. In particular, different versions of the classical spherical model (CSM) introduced by Berlin and Kac can be solved exactly for both, short [1] and long range [2,3] interactions. The spherical model (SM) was originally introduced to study ferromagnetic systems in different spatial and spin dimensions [1,3]. Unlike the mean-field approximation, in the case of spin dimensionality higher than one, the spherical model rightly yielded no ferromagnetic transition as had been observed experimentally. Moreover, its equivalence to a spin system with infinity spin dimensions has been completly established by Stanley [7]. Because of this, different versions of the SM have been recently used to investigate the physics of systems which hamiltonian can be maped out onto that of the spherical model 8 -11]. The basic idea is the compactification and replacing of too many restrictions by one spherical like condition. Thus, studying the phase transitions and the phase diagram of a spin ferromagnet or antiferromagnet using a particular version of the SM is of physical relevance.

In this paper we investigate the phase behavior of a generalized spherical version of the Blume-Emery-Griffiths (BEG) model $\mid \boldsymbol{| l}$. This is obtained from the discrete three states spin one ferromagnet or antiferromagnet and, as in the original BEG model, we include magnetic spin interactions as well as quadrupole interactions. The BEG model was introduced to understand qualitatively the phase separation, and the $\lambda$ transition of ${ }^{3} \mathrm{He}-{ }^{4} \mathrm{He}$ mixtures. It has also been applied to study magnetic systems with competing interactions [5] or to describe the qualitative phase behavior of a microemulsion [6].

By introducing a transformation that maps out the original BEG model hamiltonian onto that of the SM we are able to investigate the conditions under which phase transitions may occur. Proceeding as in the investigation of the phase transitions of the CSM we study the properties of the saddle points of the integrand of the partition function.

The main findings of this paper are the following: (1) There is no phase transition at all in $d=1,2$ spatial dimensions for both, the FM and AFM systems. This result agrees well with that obtained for the CSM. (2) For $d=3$ dimensions and zero external magnetic field, we obtain the usual paramagnetic-ferromagnetic(antiferromagnetic) [PMFM(AFM)] phase transition if the FM (AFM) interactions dominate over the quadrupole interactions. On the opposite side, when the quadrupole interactions dominate over the magnetic exchange couplings no phase transition occurs. This result is consistent with the limit of zero magnetic exchange interactions. However, in the intermediate regime, when both, quadrupole and exchange interactions are relevant, there appears at low temperatures, a novel and intriguing reentrant phase transition, in addition to the regular high temperature one. That is, the FM or AFM ordering takes place at intermediate temperatures, since at high and low temperatures a PM phase sets in. (3) For a FM system in a nonzero external uniform magnetic field and $d=3$ spatial dimensions, the transition to a spontaneously magnetized state is destroyed, so no phase transition occurs. Nonetheless, for AFM exchange interactions in a uniform magnetic field we find a scenario similar to that described in (2) for $H=0$, except that now the critical temperatures are now functions of $H$. More importantly, the domain of parameters where the reentrant phase transition happens narrows as the external magnetic field increases and, at the end, the transition completely disappears above a certain critical value $H_{c 1}$. Increasing further 
the external magnetic field yields a second critical field value $H_{c 2} \approx 2 H_{c 1}$, above which the AFM phase is supressed.

The layout of the remainder of this paper is the following: in section II we introduce the BEG model hamiltonian and map it onto a generalized version of the spherical model. Then, we investigate the general conditions under which phase transitions may take place. In section III we consider the case of short range interactions and study the different scenarios that may occur in the system. A novel global spherical condition that is fully equivalent to the SM and valid even for low spin dimensionality is introduced in section $\mathbb{I V}$. Finally, in section $\mathrm{V}$ we present a summary of our findings and the conclusions.

\section{THE MODEL}

Let us start with a BEG type model hamiltonian with spins $S_{\vec{R}}=1$ located at each site of a $d$-dimensional rectangular lattice. Thus, each spin has three different states namely, $S_{\vec{R}}^{\mathrm{z}}=0, \pm 1$. It is natural to assume that the spins interact with each other through the pair potential $V\left(\vec{R}-\vec{R}^{\prime}\right)$ and with an effective external magnetic field $H=M_{0} H_{0}$, where $H_{0}$ is a "true external field" and $M_{0}$ is the magnetic moment produced by the states whith $S_{\vec{R}}^{\mathrm{z}}= \pm 1$. According to these statements the Hamiltonian that defines the model is:

$$
\mathcal{H}=E \sum_{\vec{R}} S_{\vec{R}}^{2}+\sum_{\vec{R}, \vec{R}^{\prime}} V\left(\vec{R}-\vec{R}^{\prime}\right) S_{\vec{R}} S_{\vec{R}^{\prime}}+H \sum_{\vec{R}} S_{\vec{R}}
$$

where the second sum may run over all nearest neighbors or over all pairs of spins. The first term in $\mathcal{H}$ has been introduced in order to have the possibility of quadrupolar ordering, as suggested in reference [4], in addition to the magnetic ordering associated with the second term. In absence of an external magnetic field the competition between these two types of ordering is measured by the parameter $E$, which can be interpreted as the energy difference between the states with $S_{\vec{R}}^{\mathrm{z}}= \pm 1$ and $S_{\vec{R}}^{\mathrm{z}}=0$. With the aim at mapping this model hamiltonian onto that of the spherical model, we introduce the Ising-like variables $a_{\vec{R}}= \pm 1$ and $b_{\vec{R}}= \pm 1$ that are related to the original spin variables by,

$$
S_{\vec{R}}=\frac{1}{2}\left(a_{\vec{R}}+b_{\vec{R}}\right) .
$$

Under this transformation the state $S_{\vec{R}}=0$ has been double weighted since it can now be obtained from the states $a_{\vec{R}}=-b_{\vec{R}}=1$ and $a_{\vec{R}}=-b_{\vec{R}}=-1$. To compensate this "degeneration" we introduce, in an ad hoc manner, the additional entropy term, $\mathcal{H}_{\mathcal{S}}=-\frac{1}{4} T \ln 2 \sum_{\vec{R}} S_{\vec{R}}^{2}$, in units in which the Boltzmann constant $k_{B}$ is equal to unity. In terms of these Ising-like new variables and including the entropy term the hamiltonian $\mathcal{H}$ becomes:

$$
\begin{aligned}
\mathcal{H} & =\frac{1}{4}(E-T \ln 2) \sum_{\vec{R}}\left(a_{\vec{R}}+b_{\vec{R}}\right)^{2}+\frac{1}{4} \sum_{\vec{R}, \vec{R}^{\prime}} V\left(\vec{R}-\vec{R}^{\prime}\right)\left(a_{\vec{R}}+b_{\vec{R}}\right)\left(a_{\vec{R}^{\prime}}+b_{\vec{R}^{\prime}}\right) \\
& +\frac{H}{2} \sum_{\vec{R}}\left(a_{\vec{R}}+b_{\vec{R}}\right) .
\end{aligned}
$$

To be able to study analytically the properties of the model we have to make contact with the classical spherical model [1,3]. So, we replace the discrete Ising-variables by continuous 
ones, that is, we let the "new" spin variables to vary in the interval, $-\infty<a_{\vec{R}}, b_{\vec{R}}<+\infty$, obeying the spherical conditions:

$$
\sum_{\vec{R}} a_{\vec{R}}^{2}=\mathcal{N}, \text { and } \sum_{\vec{R}} b_{\vec{R}}^{2}=\mathcal{N}
$$

where $\mathcal{N}$ is the total number of spins in the system. Hence, the hamiltonian, Eqn. (3) together with the last two restrictions can be considered as the "generalized spherical version" of the BEG hamiltonian, Eqn. (1). The reason of this name is that we have included the additional entropy term that depends explicitly on temperature in the quadrupole interaction part of the Hamiltonian. This term does not appear in the original BEG model and will play a key role in the results we describe in what follows.

Taking into account these spherical conditions on the "new" Ising-like variables, one can write the partition function as follows:

$$
\mathcal{Z}=\left\{\prod_{\{\vec{R}\}} \int_{-\infty}^{\infty} d a_{\vec{R}} \int_{-\infty}^{\infty} d b_{\vec{R}}\right\} \exp \left(-\frac{\mathcal{H}}{T}\right) \delta\left(\mathcal{N}-\sum_{\vec{R}} a_{\vec{R}}^{2}\right) \delta\left(\mathcal{N}-\sum_{\vec{R}} b_{\vec{R}}^{2}\right) .
$$

It is important to point out that the transformation from discrete Ising spin variables to the continuous spin ones is not quite correct since there are "wrong" configurations in which there are spin variables that may take values different from \pm 1 . Nonetheless, such "slight incorrect transformation" allows one to solve analitycally two classical problems: the 3D Ising Model [1, 3], and more importantly, the 3D Heisenberg model [7], in addition to other more recent contemporary physical problems [8 11]. In section IV we address this shortcoming and propose an absolutely correct global spherical condition that is even valid for systems of low spin dimensionality, in particular for $\vec{S}_{\vec{R}}=1$. For the time being we proceed as in the CSM, that is, we use the integral representation of $\delta$-function to rewrite the partition function as:

$$
\mathcal{Z}=\int \frac{d s_{1} d s_{2}}{(2 \pi i)^{2}} \exp \left(\mathcal{N}\left(s_{1}+s_{2}\right)\right) \tilde{\mathcal{Z}}\left(s_{1}, s_{2}\right)
$$

where

$$
\tilde{\mathcal{Z}}=\left\{\prod_{\vec{R}} \int_{-\infty}^{\infty} d a_{\vec{R}} \int_{-\infty}^{\infty} d b_{\vec{R}}\right\} \exp \left(-\frac{\tilde{\mathcal{H}}\left(s_{1}, s_{2}\right)}{T}\right)
$$

and

$$
\tilde{\mathcal{H}}=T s_{1} \sum_{\vec{R}} a_{\vec{R}}^{2}+T s_{2} \sum_{\vec{R}} b_{\vec{R}}^{2}+\mathcal{H}
$$

where $\mathcal{H}$ is given in Eqn. (11), with the $\vec{S}_{\vec{R}}$ replaced by $\frac{1}{2}\left(\vec{a}_{\vec{R}}+\vec{b}_{\vec{R}}\right)$. In the framework of the CSM the effective hamiltonian $\tilde{\mathcal{H}}$ is a non-diagonal symmetric bilinear form in the new Ising-like variables. To be able to integrate Eqn. (7) one has to diagonalize $\tilde{\mathcal{H}}$ by means of a Fourier transformation of the spin variables. In doing so one gets,

$$
\mathcal{Z}=\pi^{\mathcal{N}} \int \frac{d s_{1} d s_{2}}{(2 \pi i)^{2}} \exp \left(\mathcal{N} \gamma\left(s_{1}, s_{2}\right)\right)
$$


where

$$
\gamma\left(s_{1}, s_{2}\right)=s_{1}+s_{2}-\frac{1}{2 \mathcal{N}} \sum_{\vec{q}} \ln \left(s_{1} s_{2}+\left(s_{1}+s_{2}\right) \frac{\alpha(\vec{q})}{T}\right)+\frac{H^{2}}{16 T^{2}} \frac{s_{1}+s_{2}}{\frac{\alpha(0)}{T}\left(s_{1}+s_{2}\right)+s_{1} s_{2}},
$$

and

$$
\alpha(\vec{q})=\frac{1}{4}\left[E-T \ln 2+\sum_{\vec{R}} V(\vec{R}) \exp (-i \vec{q} \vec{R})\right]
$$

where the summation in Eqn. (10) is carried out over the first Brillouin zone (BZ). The next step would be the evaluation of the partition function, however, instead of doing it we proceed as in the study of the CSM. That is, we find the saddle points of $\mathcal{Z}$ and study its temperature behavior since it contains the complete information of the possible phase transitions of the system. Nonetheless, it is important to note that the evaluation of the partition function by the steepest descendent method becomes exact in the thermodynamic limit $\mathcal{N} \rightarrow \infty$, as in the CSM. The saddle points of the integrand of $\mathcal{Z}$ are determined by the following equations:

$$
\begin{aligned}
\frac{\partial \gamma}{\partial s_{1}}=0 & =1-\frac{1}{2\left(s_{1}+s_{2}\right)}-\frac{1}{2 \mathcal{N}} \frac{s_{2}^{2}}{s_{1}+s_{2}} \sum_{\vec{q}} \frac{1}{s_{1} s_{2}+\frac{\alpha(\vec{q})}{T}\left(s_{1}+s_{2}\right)} \\
& -\frac{H^{2}}{16 T^{2}} \frac{s_{2}^{2}}{\left(\frac{\alpha(0)}{T}\left(s_{1}+s_{2}\right)+s_{1} s_{2}\right)^{2}}, \\
\frac{\partial \gamma}{\partial s_{2}}=0 & =1-\frac{1}{2\left(s_{1}+s_{2}\right)}-\frac{1}{2 \mathcal{N}} \frac{s_{1}^{2}}{s_{1}+s_{2}} \sum_{\vec{q}} \frac{1}{s_{1} s_{2}+\frac{\alpha(\vec{q})}{T}\left(s_{1}+s_{2}\right)} \\
& -\frac{H^{2}}{16 T^{2}} \frac{s_{1}^{2}}{\left(\frac{\alpha(0)}{T}\left(s_{1}+s_{2}\right)+s_{1} s_{2}\right)^{2}} .
\end{aligned}
$$

These equations are symmetric in the variables $s_{1}$ and $s_{2}$. Substracting Eqn. (13) from Eqn. (12) and taking into account that $\gamma\left(s_{1}, s_{2}\right)$ has to be an analytic function at the saddle point, one gets the condition $s_{1}=s_{2}=s$. Substituting this in either, Eqns. (12) or (13), we obtain the equation that yields the saddle points of the integrand of the partition function, namely

$$
\psi(s)=\frac{1}{\mathcal{N}} \sum_{\vec{q}} \frac{1}{s+2 \frac{\alpha(\vec{q})}{T}}+\frac{H^{2}}{4 T^{2}} \frac{1}{\left(s+2 \frac{\alpha(0)}{T}\right)^{2}}+\frac{1}{s}=4 .
$$

To investigate the existence of phase transitions in the model, we should recall that, as in the CSM, the existence of a saddle point is associated with the setting in of a disordered phase, PM phase in this case, while the absence of a saddle point is related to the realization of an ordered phase, FM or AFM. The critical temperature is obtained by studying the evolution of the saddle point as a function of temperature. To be more 
precise, the phase transition occurs just when the saddle point dissapears. As we will see below there is a such transition in some region of the parameters space $(E, J, H)$, nonetheless, there is another region in the parameters space where a saddle point does exist for any temperature, indicating the absence of a phase transition in such region. For instance, one can easily show that for a nonzero external magnetic field and if the ferromagnetic interactions between spins satisfy the condition $\min _{\vec{q}} \alpha(\vec{q})=\alpha(0)$, then a saddle point exists in the whole parameter space and therefore no phase transition takes place. However, for antiferromagnetic interactions one gets $\min _{\vec{q}} \alpha(\vec{q})<\alpha(0)$ and a more careful and detailed analysis should be carried out to obtain the corresponding phase diagram.(See subsection [IIB). In the following section we apply our general model hamiltonian to study a system with short range interactions.

\section{PHASE TRANSITIONS IN A FERROMAGNETIC AND AN ANTIFERROMAGNETIC SYSTEMS WITH SHORT-RANGE INTERACTIONS.}

\section{A. Zero external magnetic field.}

Let us consider a magnetic system with short range interactions, that is, $V\left(\vec{R}-\vec{R}^{\prime}\right)=$ $-J$ for nearest neighbors $\vec{R}, \vec{R}^{\prime}$, and zero otherwise. In this case Eqn. (11) becomes:

$$
\alpha(\vec{q})=\frac{1}{4}\left(E-T \ln 2-2 J \sum_{j=1}^{d} \cos q_{j}\right),
$$

where $d$ is the spatial dimensionality, $\vec{q}=\left(a_{1} q_{1}, a_{2} q_{2}, \ldots ..\right)$ is a dimensionless vector in the reciprocal space and $a_{j}$ is the lattice constant along the $j$ direction. Setting $H=0$ in Eqn. (14), the function $\psi(s)=\psi_{0}(s)$ can be rewritten as

$$
4=\psi_{0}(s)=\frac{1}{s}+\frac{T}{J} \frac{1}{(2 \pi)^{d}} \int_{B . Z .} d \vec{q} \frac{1}{\frac{T}{J}\left(s+\frac{E}{2 T}-\frac{1}{2} \ln 2\right)-\left(\sum_{j=1}^{d} \cos q_{j}\right)},
$$

here the integration is carried out in the first Brillouin zone, that is, in the range $-\pi<$ $q_{j}<\pi$ for each $j$. As we know and according to the definition of the interaction potential, $J>0$, for ferromagnetic interactions while $J<0$, for antiferromagnetic interactions. Notice that in the framework of our model the phase diagram in the $E$ versus $J$ plane for the antiferromagnet $(J<0)$ is obtained from the reflection about the line $J=0$ of the phase diagram of the ferromagnet. Indeed, by introducing a shift in the wave vectors in $q$-space, that is, by replacing $\vec{q}$ by $\vec{q}^{*}=\left(q_{1}-\pi, q_{2}-\pi, \ldots\right)$ one can show that $J \sum \cos q_{j}=-J \sum \cos q_{j}^{*}$. Therefore, Eqn. (16) for the antiferromagnetic system results in that corresponding to a ferromagnet. The only difference being the critical value $\vec{q}=\vec{q}_{\mathrm{m}}$ at which $\alpha(\vec{q})$ attains its minimum value. For a ferromagnet this minimum is reached at $\vec{q}_{\mathrm{m}}=0$ while for an antiferromagnet the minimum occurs at $\vec{q}_{\mathrm{m}}=(-\pi,-\pi, \ldots)$. In term of the orientations of spins this is equivalent to say that nearest-neighbors spins try to align parallel for ferromagnetic interactions or anti-parallel for antiferromagnetic interactions. Thus, all particularities of the phase transition of the antiferromagnet are the same as those for the ferromagnet. Bearing this in mind we carry out a detailed analysis of the 
phase diagram for a ferromagnet and extend directly the results for an antiferromagnet by carrying out a reflection about $J=0$.

The next step is to examine the properties of $\psi_{0}(s)$ for a ferromagnet since they determine the phase transitions in the model under investigation. It is easy to see that $\psi_{0}(s)$ decreases monotonically and is analytic in the interval $s_{0}(T)<s \leq+\infty$, where $s_{0}$ is defined as,

$$
s_{0}=\max \left(0, \frac{1}{2} \ln 2-(E-2 d J) / 2 T\right) .
$$

The behavior of the function $\psi_{0}(s)$ is such that it can take values from zero when $s \rightarrow \infty$ up to a nonzero value $\psi_{0}\left(s_{0}\right)$. Its behavior is shown in Fig. 1, where we have indicated the positions of $s_{0}$ (points $\mathbf{B}$ and $\mathbf{A}$ ) at two temperatures $T_{1}$ and $T_{2}$ that are below and above the critical temperature $T_{c}$, respectively. The point $\mathbf{R}$ represents the saddle point which existence is related to the set in of the disordered phase. Thus, at $T=T_{2}$ (point $\mathbf{P}$ and dot dashed line), $\psi_{0}\left(s_{0}\right)>4$, and Eqn. (16) has a root, indicative of the existence of a saddle point and a paramagnetic phase. In the opposite case, at $T=T_{1}$ (point $\mathbf{Q}$ and solid line), $\psi_{0}\left(s_{0}\right)<4$, and Eqn. ( 16 ) has no root yielding no phase transition to the paramagnetic phase. Hence it is necesary to analyze the behavior of $\psi_{0}\left(s_{0}\right)$ as a function of temperature in the parameters space $(E, J)$ to be able to construct the phase diagram. First of all, we have to study the general conditions for which the inequality $\psi_{0}\left(s_{0}\right)<4$, responsible for the ferromagnetic phase, is valid. Thus, in the region of parameters $(E$, $J, T)$ where $s_{0}=0$, the function $\psi_{0}\left(s_{0}\right) \rightarrow \infty\left(\psi_{0}\left(s_{0}\right)>4\right)$ and a paramagnetic phase exists. Nonetheless, when $s_{0}>0$ it is necessary to analyse the convergence of the integral for $s=s_{0}$ in Eqn. (16). In doing so we substitute $s=s_{0}$ and expand $\cos q_{j} \approx 1-q_{j}^{2} / 2$, yielding,

$$
\int_{B . Z .} d \vec{q} \frac{1}{\frac{T}{J}\left(s_{0}+\frac{E}{2 T}-\frac{1}{2} \ln 2\right)-\left(\sum_{j=1}^{d} \cos q_{j}\right)} \sim \int_{B . Z .} \frac{q^{d-1} d q}{q^{2}},
$$

where we have omited an irrelevant constant numerical factor. The last integral converges if the spatial dimension $d$ is such that, $d-3>-1$, i.e. for spatial dimensionality $d>2$. This means that for $d \leq 2, \psi_{0}\left(s \rightarrow s_{0}\right) \rightarrow \infty$ yielding a saddle point for any values of $E, J$ and $T$, and no phase transition takes place for lower dimensional systems. A more detailed analysis has to be carried out for three dimensions because the integral (18) does converge and can be evaluated as [1],

$$
\frac{1}{(2 \pi)^{3}} \int \frac{d \vec{q}}{3-\sum_{j=1}^{3} \cos q_{j}} \approx 0.50541=I_{\mathrm{cr}} .
$$

Introducing this result in Eqn. (16) one obtains, for $s_{0}(T)>0$,

$$
\psi_{0}\left(s_{0}(T), T\right)=\frac{T}{J} I_{\mathrm{cr}}+\frac{2 T}{T \ln 2-E+6 J}
$$

In this case we find (see Fig. 2) that there are regions in the parameter space $(E, J, T)$ where $\psi_{0}\left(s_{0}\right)<4$, i.e. the saddle point disappears. This happens for temperatures below a certain critical temperature, $T_{c}$, indicating the transition to the ferromagnetic 
(antiferromagnetic) state. A more detailed analysis of $\psi_{0}\left(s_{0}(T), T\right)$ shows that there are, actually, two ranges of parameters to be considered: (1) $E-6 J<0$ in which case $s_{0}(T)$ goes from $+\infty$ at $T=0$ up to $\frac{1}{2} \ln 2$ at $T=\infty$, and (2) $E-6 J>0$ for which $s_{0}(T)$ goes from zero at $T=0$ up to $\frac{1}{2} \ln 2$ at $T=\infty$.

Let us first consider the behavior of $\psi_{0}\left(s_{0}(T), T\right)$ as a function of temperature when $E-6 J<0$. In this domain of parameters $s_{0}>0$ for any $T$ and Eqn. (19) is always valid. At very low temperatures $\psi_{0}\left(s_{0}(T), T\right)<4$ and at $T=0, \psi_{0}\left(s_{0}\right)$ goes down to zero, implying that there is no saddle point, thus the system settles down in a ferromagnetic state. At higher temperatures, the function $\psi_{0}\left(s_{0}(T), T\right)$ increases almost linearly with temperature and therefore $\psi_{0}\left(s_{0}\right)$ crosses over the line $\psi_{0}\left(s_{0}\right)=4$, at the critical temperature and the saddle point shows up, hence the system goes from the ferromagnetic (antiferromagnetic) phase to the paramagnetic one. Thus, this region of parameters yields one critical temperature that corresponds to the usual PM-FM (or PM-AFM) phase transition. On the other hand, in the range of parameters where $E-6 J>0$ the behavior of $\psi_{0}\left(s_{0}(T), T\right)$ is different. For $T<(E-6 J) / \ln 2$, the function $\psi_{0}\left(s_{0}\right)$ diverges since $s_{0}=0$. For $s_{0}(T)>0$, the function $\psi_{0}\left(s_{0}(T)\right)$ decreases asymptotically and quite rapidly from $\infty$ at $T=(E-6 J) / \ln 2$ until it reaches a minimum at the temperature $T^{*}=T^{*}(E, J)$, given by

$$
T^{*}=\frac{1}{\ln 2}\left(E-6 J+\sqrt{\frac{2 J(E-6 J)}{I_{\mathrm{cr}}}}\right),
$$

and afterwards, it begins to increase. This behavior is shown in detail in Fig. 2. It is obvious that as soon as $\psi_{0}\left(s_{0}\left(T^{*}\right), T^{*}\right)>4$ then $\psi_{0}\left(s_{0}(T), T\right)>4$ for any temperature $T$ greater than $T^{*}$ and the paramagnetic phase is only present, therefore, no phase transition occurs. This is what happens when $\frac{E}{J}>6+x_{c}$ for any value of $T$, where, the value of $x_{c}$ is given by $x_{c}=\left(1 / I_{c r}\right)(2 \sqrt{\ln 2}-\sqrt{2})^{2} \approx 0.124$. The corresponding curves $\psi_{0}\left(s_{0}(T), T\right)$ that fullfil this condition are shown in Fig. 2. However, if $6<\frac{E}{J}<6+x_{c}$ then $\psi_{0}\left(s_{0}\left(T^{*}\right), T^{*}\right)<4$, and $\psi_{0}\left(s_{0}(T), T\right)$ intersects the line $\psi=4$ in two points, yielding two critical temperatures: $T_{c}^{+}$, the branch where the PM-FM (PM-AFM) phase transition occurs, and $T_{c}^{-}$, the branch at which the system goes back to the paramagnetic phase; so a reentrant phase transition takes place. These critical temperatures are given by

$$
T_{c}^{ \pm}=\frac{(E-6 J) I_{\mathrm{cr}}+4 J \ln 2-2 J \pm \mathcal{D}}{2 I_{\mathrm{cr}} \ln 2},
$$

with

$$
\mathcal{D}=\left[\left((E-6 J) I_{\mathrm{cr}}+4 J \ln 2-2 J\right)^{2}-16(E-6 J) I_{\mathrm{cr}} J \ln 2\right]^{1 / 2} .
$$

From these results we can state that in the temperature range $T_{c}^{-}<T<T_{c}^{+}$a magnetic ordered state takes place, whereas outside of this interval, i.e. at lower $T<T_{c}^{-}$and higher $T>T_{c}^{+}$temperatures, a paramagnetic state settles in. Translating these results in the parameter domain we can say that, the region $6<\frac{E}{J}<6+x_{c}$ where the reentrant phase transition occurs, separates out the region $E-6 J<0$ where the usual PM-FM (PM-AFM) phase transition happens, from the region $6+x_{c}<\frac{E}{J}$ where there is no phase transition at all. 
To make more intuitive and explicit the above results we have plotted the two dimesional phase diagram $\frac{T_{c}^{ \pm}}{J}$ versus $\frac{E}{J}$ in figure 3 . The solid line represents the critical temperature $T_{c}^{+}$that corresponds to the usual high temperature single phase transition while the dashed line represents the novel and intriguing low temperature reentrant phase transition $T_{c}^{-}$. These lines have also been plotted in a larger scale in the inset, where the two branches are clearly seen. The two critical temperatures become gradually closer to each other with increasing $E / J$ until the ordered phase dissapears completely.

\section{B. Nonzero uniform magnetic field.}

In this subsection we investigate how the phase diagram of the generalized BEG model changes in the presence of an homogeneous magnetic field in three spatial dimensions. In the case of ferromagnetic interactions the application of an external magnetic field terminates the transition to the state with a spontaneous magnetization since a saddle point appears for any values of $E, J$ and $T$, as it just happens for the CSM [1]. That is, the external magnetic field is a symmetry breaking field that produces a magnetized state that always yields a saddle point in the integrand of the partition function for any values of the parameters. Because of this, an additional analysis should be done to study the thermodynamics of the present model, for instance, one can study the behavior of the magnetic susceptibility, as was done in [1] for the CSM. For the time being, it is the purpose of the present paper to study the phase diagram and leave the study of the thermodynamics for a forthcoming paper [12]. Let us now focus our attention on the case of antiferromagnetic interactions. Basically, we will study the circumstances under which the saddle point dissapears in the presence of an homogeneous magnetic field, i.e. when the antiferromagnetic ordering sets in. Using Eqn. (14) and the results of section III A it is easy to show that $\psi(s)$ is an analytic function that decreases monotonically in the interval $s_{0}<s<\infty$, where $s_{0}$ is given by Eqn. (17). To be able to use the results obtained for an antiferromagnet in a zero magnetic field, in what follows we will denote the modulus of the AFM exchange interaction as $J>0$ since the minus sign has already been included as a shift in the reciprocal space vectors, that is, we have replaced $\vec{q}$ by $\vec{q}^{*}$. Henceforth, in analogy with the case of zero magnetic field, whenever $\psi\left(s_{0}(T), T, H\right)>4$, a saddle point does exist and it yields a paramagnetic phase, whereas in the opposite case, $\psi\left(s_{0}(T), T, H\right)<4$, the system settles in an antiferromagnetic state, where the function $\psi\left(s_{0}(T), T, H\right)$ is given by

$$
\psi\left(s_{0}(T), T, H\right)=\psi_{0}\left(s_{0}(T), T\right)+\left(\frac{H}{12 J}\right)^{2}=\frac{T}{J} I_{\text {cr }}+\frac{2 T}{T \ln 2-E+6 J}+\left(\frac{H}{12 J}\right)^{2},
$$

To obtain this equation we have made the following substitution in Eqn. (14),

$$
H^{2} /\left(4 T^{2}\left(s_{0}+2 \alpha(\vec{q}=0) / T\right)^{2}\right)=\left(\frac{H}{12 J}\right)^{2} .
$$

As in the previous subsection, the equation $\psi_{0}\left(s_{0}\left(T_{c}\right), T_{c}, H\right)=4$ determines the temperature at which the phase transition occurs. From this very last condition one can rewrite Eqn. (23) as ,

$$
\psi_{0}\left(s_{0}\left(T_{c}\right), T_{c}\right)=4-\left(\frac{H}{12 J}\right)^{2}
$$


where $\psi_{0}\left(s_{0}(T), T\right)$ is the function that we have carefully studied for the ferromagnetic and antiferromagnetic systems in abscence of an external field. Thus, one obtains $T_{c}(H)$ by looking at the intercept of the curves $\psi_{0}\left(s_{0}\right)$ shown in Fig. 2, with the lines $4-(H / 12 J)^{2}$. The resulting plots are shown in Fig. 6 for the following values of the reduced magnetic field, $\frac{H}{J}$ : (a) 2, (b) 8, (c) 16 and (d) 20. One immediately sees that in the parameter region $E-6 J<0$, there is only one root of the equation $\psi\left(s_{0}\left(T_{c}\right), T_{c}, H\right)=4$, what signals the usual transition to the antiferromagnetic phase. The critical temperature of this transition, $T_{c}(H)$, that is now a function of the external magnetic field, diminishes as the field intensity increases and becomes equal to zero at the critical field value $H_{c 2}=24 \mathrm{~J}$. Thus, the phase transition dissapears for fields such that $H>H_{c 2}$, and the paramagnetic state settles in at all temperatures, as one naturally would expect, since the antiferromagnetic ordering is broken by higher external field intensities. We have represented this critical field value by $H_{c 2}$ because of, as we will show below, there is another critical field value $H_{c 1} \approx 0.5 H_{c 2}$ at which the reentrant phase transition dissapears.

On the other hand, in the parameter region where $E-6 J>0$ there are two possibilities: (i) the reentrant AFM-PM phase transition or (ii) the absence of a phase transition at all. The domain of parameters where the reentrant transition takes place is restricted by the inequalities, $E-6 J>0$ and $\psi_{0}\left(s_{0}\left(T^{*}\right), T^{*}\right)<4-(H / 12 J)^{2}$, where $T^{*}$ is defined in Eqn. (20). These two relations can be reexpressed in the form: $6<E / J<6+x_{c}(H)$, with $x_{c}(H)$ given by

$$
x_{c}=\frac{\left(2 \sqrt{\ln 2} \sqrt{1-\left(\frac{H}{H_{c 1}}\right)^{2}}-\sqrt{2}\right)^{2}}{I_{\mathrm{cr}}} .
$$

The two critical temperature branches of the phase transitions are the roots of the equation $\psi\left(s_{0}\left(T_{c}\right), T_{c}, H\right)=4$, and are given by

$$
T_{c}^{ \pm}=\frac{(E-6 J) I_{\mathrm{cr}}-2 J+J\left(4-(H / 12 J)^{2}\right) \ln 2 \pm \mathcal{D}(H)}{2 I_{\mathrm{cr}} \ln 2}
$$

where

$$
\mathcal{D}=\sqrt{\left((6 J-E) I_{\mathrm{cr}}+2 J-J\left(4-\left(\frac{H}{12 J}\right)^{2}\right) \ln 2\right)+4 I_{\mathrm{cr}} J(6 J-E)\left(4-\left(\frac{H}{12 J}\right)^{2}\right) \ln 2 .}
$$

Evidently, the reentrance phenomenon is suppressed by the external field when it exceeds the critical value $H_{c 1}$ defined as the root of the equation $x_{c}\left(H_{c 1}\right)=0$. This leads to the critical field value,

$$
H_{c 1}=H_{c 2} \frac{\sqrt{4-2 / \ln 2}}{2}=0.52788 H_{c 2} \approx \frac{H_{c 2}}{2}
$$

These results allow us to construct the phase diagram $\left(\frac{T}{J}\right.$ versus $\left.\frac{E}{J}, \frac{H}{J}\right)$ which, for the sake of clarity, is shown in two parts, Figs. 5 and 6. The former shows the region in three dimensional space where there is a single transition, while the latter corresponds to the region where the reentrance phenomenon takes place. That is, the whole half-plane $\left(\frac{E}{J}, \frac{H}{J}\right)$ 
splits into three regions, the first one corresponds to the "single transition" characterized by the PM-AFM phase transition, while the "reentrant AFM-PM phase transition" occurs in the second region. The third region corresponds to the domain where there is no phase transition at any temperature and any values of the coupling parameters $E$ and $J$.

\section{EXACT GLOBAL SPHERICAL CONDITION.}

One of the main shortcomings of SM approach, that we have used here, Eqn. (四), is that it fails when spatial and spin dimensionality are low. For instance, it is known that in two dimensions the CSM approximation for an Ising ferromagnet does not describe the correct transition scenario. Nonetheless, for spin dimensions greater than one, the CSM approximation works quite well. In fact, it is exact in the limit of infinite spin dimensionality [7]. The main drawback of the classical spherical condition: $\sum_{\vec{R}}\left(a_{\vec{R}}^{2}-1\right)=0$ is that it holds not only for "right configurations", $a_{\vec{R}}= \pm 1$, but it also does for the "wrong configurations" where some of the spin variables are $a_{\vec{R}}<1$ while others are $a_{\vec{R}}>1$.

So, in trying to fix up this shortcoming we replace the usual spherical condition by the "general global" one, namely

$$
\sum_{\vec{R}}\left(a_{\vec{R}}^{2}-1\right)^{2}=0
$$

This sum is equal to zero only for the "right configurations" $a_{\vec{R}}= \pm 1$. Therefore, the model with continuously varying spin variables plus the "global spherical condition", Eqn. (29), are equivalent to the original spin one BEG hamiltonian. Note that we may also use the general global condition $\sum_{\vec{R}} f\left(a_{\vec{R}}^{2}-1\right)=0$ where $f(x)>0$ for $x \neq 0$ and $f(0)=0$, however, for definiteness and simplicity one can use Eqn. (29). This condition can be expressed in a form slightly closer to the usual spherical condition as follows:

$$
\mathcal{N}=\sum_{\vec{R}} a_{\vec{R}}^{2}+\sum_{\vec{R}}\left(a_{\vec{R}}^{2}-1\right)\left(a_{\vec{R}}^{2}-2\right) .
$$

With this "new global condition" we can write a new effective hamiltonian which in turn defines the partition function $\tilde{\mathcal{Z}}\left(s_{1}, s_{2}\right)$ (see, Eqns. (7) and (8)):

$$
\tilde{\mathcal{H}}\left(s_{1}, s_{2}\right)=\tilde{\mathcal{H}}_{0}+T s_{1} \sum_{\vec{R}}\left(a_{\vec{R}}^{2}-1\right)\left(a_{\vec{R}}^{2}-2\right)+T s_{2} \sum_{\vec{R}}\left(b_{\vec{R}}^{2}-1\right)\left(b_{\vec{R}}^{2}-2\right),
$$

where $\tilde{\mathcal{H}}_{0}$ is the usual spherical effective hamiltonian defined by Eqn. (8). So one can rewrite the new effective hamiltonian as the usual spherical hamiltonian plus an additional interaction term $U\left(s_{1}, s_{2}\right)$, that is: $\tilde{\mathcal{H}}\left(s_{1}, s_{2}\right)=\tilde{\mathcal{H}}_{0}+U\left(s_{1}, s_{2}\right)$. Note that this new effective hamiltonian is much more complicated than the one we have studied in this paper and, unfortunately, the interaction $U\left(s_{1}, s_{2}\right)$ cannot be considered perturbatively because it lacks of a small parameter. However, our propossal allows us, in principle, to improve the classical spherical model for a system with lower spin and space dimensionalities. The problem of incorporating the $U(s)$ interaction into the complete solution is a difficult task and it is the subject of current research. 


\section{SUMMARY AND CONCLUSIONS}

In this paper we have introduced and studied the phase diagrams of a generalized spherical version of the BEG model considering ferromagnetic as well as antiferromagnetic interactions. The model hamiltonian involves terms representative of quadrupole interactions and magnetic spin interactions in zero and nonzero external magnetic field. We have shown explicitly that in the short range interactions limit and zero external magnetic field the model presents no phase transition in one and two spatial dimensions. However, in three dimensions it undergoes a regular PM-FM(AFM) transition at nonzero temperature. This happens in the case when the magnetic interactions dominate over the quadrupole ones. Nonetheless, in the range of parameters where quadrupole and magnetic interactions are relevant we obtain a low temperature reentrant FM(AFM)-PM phase transition, in addition to the conventional PM-FM(AFM) phase transition at higher temperatures. These phase transitions are absent in the strong quadrupole interaction regime. On the other hand, when there is an external magnetic field there is no phase transition for the ferromagnet in three dimensions, since there appears a permanent magnetized state that terminates the PM-FM transition. This result appears to be similar to the one obtained in the CSM [1], and it is a motivation to further investigate the thermodynamics of the present model [12]. However, in the case of antiferromagnetic interactions and nonzero magnetic field we also get the reentance phenomenon whenever the magnetic spin and quadrupole interactions compete between them and always that $H<H_{c 1} \approx 12 J$. If $H_{c 1} \leq H$ the reentrant phase transition dissapears. We also find a second critical value of the external magnetic field $H_{c 2}$, above which the PM-AFM phase transition dissapears. Finally, we have introduced a "novel global spherical condition" that makes our approach valid even for low spin dimensionality. However, this led us to a more complicated hamiltonian which phase diagram is under current investigation.

\section{ACKNOWLEDGEMENTS}

This work has been supported by CONACYT-MEXICO grant No. 25298-E. 


\section{REFERENCES}

[1] T. H. Berlin and M. Kac, Phys. Rev. 86, 821 (1952).

[2] G. S. Joyce, Phys. Rev. 146, 349 (1966).

[3] A. M. Khorunzhy, B. A. Khoruzhenko, L. A. Pastur, and M. V. Shcherbina, in Phase Transitions and Critical Phenomena, edited by C. Domb and J. Lebowitz (Academic, New York, 1992), Vol. 15, p. 74.

[4] M. Blume, V. J. Emery and R. B. Griffiths, Phys. Rev. A4 (1971), 1071.

[5] P. J. Jensen, K. A. Penson and K. H. Bennemann, Phys. Rev. B35 (1987), 7306.

[6] M. Schick and W. H. Shih, Phys. Rev. Lett. 59 (1987), 1205; F. Schmid and M. Schick, Phys. Rev. E49, 494 (1994).

[7] H. E. Stanley, Phys. Rev. 176,718 (1968); ibid, Phys. Rev. 179, 570 (1969); M. Kac and C.J. Thompson, Phys. Norvegica, 5, 163 (1971).

[8] Thomas Vojta, Phys. Rev. B53, 710 (1996).

[9] B. Bergersen and Z. Rácz, Phys. Rev. Lett. 67, 3047 (1991); Chung-Yu Mou and P. B. Weichman, ibid 70, 1101 (1993); J.W. Hartman and P. B. Weichman, ibid 74, 4584 (1995); D. C. Brody, Phys. Rev. E49, 3665 (1994).

[10] T. K. Kopeć, J. V. José, Phys. Rev. B60, 7473 (1999); ibid, Phys. Rev. Lett. 84, 749 (2000).

[11] Sergey E. Savel'ev and G. Ramírez-Santiago, Bull. Am. Phys. Soc. Vol. 44 (1999), No. 1 part II, p. 1697.

[12] Sergey E. Savel'ev and G. Ramírez-Santiago, In preparation. 
FIG. 1. Behavior of $\psi$ as function of the parameter $s$ at two temperatures $T_{1}$ and $T_{2}$ below and above the critical temperature, respectively. Points $\mathbf{A}$ and $\mathbf{B}$ have coordinates $\left(s_{0}\left(T_{2}\right), 0\right)$ and $\left(s_{0}\left(T_{1}\right), 0\right)$, while the coordinates of points $\mathbf{P}$ and $\mathbf{Q}$ are $\left(s_{0}\left(T_{2}\right), \psi\right)$ and $\left(s_{0}\left(T_{1}\right), \psi\right)$, respectively. Point $\mathbf{R}$ indicates the saddle point that signals the phase transition. Note that $\psi(s)$ decreases monotonically with $s$, that is, it goes from $\psi\left(s_{0}\right)$ and goes down to zero asymptotically as $s \rightarrow \infty$.

FIG. 2. Behavior of $\psi_{0}\left(s_{0}\right)$ as function of $\frac{T}{J}$ and different values of $\frac{E}{J}$. Note that for $0<\frac{E}{J}<6$ there is only one intercept of these curves with the line $\psi_{0}=4$, as an indication of a single transition. However, for $6 \leq \frac{E}{J}<6+x_{c}$ we see a lower temperature intercept corresponding to the reentrant phase transition.

FIG. 3. Phase diagram for the ferromagnet where the reentrant transition appears at $\frac{E}{J}>6$. The letters $\mathbf{P}$ and $\mathbf{F}$ indicate the paramagnetic and ferromagnetic states, respectively. In the inset we show the part of the phase diagram corresponding to the reentrance behavior in the parameter region $6<\frac{E}{J}<6+x_{c}$. 
FIG. 4. Behavior of $\psi_{0}\left(s_{0}\right)$ versus $\frac{T}{J}$ for different values of the parameter $\frac{E}{J}$ (same values as in Fig. 22) and different values of the external magnetic field.(a) $\frac{H}{J}=2$ (b) $\frac{H}{J}=8$, (c) $\frac{H}{J}=16$, and (d) $\frac{H}{J}=20$. Notice that for $E / J<6$, where a single transition occurs, the critical temperatures $T_{c}(H)$, decrease as the magnetic field increases. On the other hand, for curves $\psi(s)$, with $6<\frac{E}{J}<6+x_{c}(H)$, there are two transitions, the high temperature one and the one corresponding to the reentrance to the paramagnetic state, panels (a) and (b), while in panels (c) and (d) there is no phase transition at all, because of the fact that $\frac{H}{J}>\frac{H_{c}}{J}$.

FIG. 5. Three dimensional phase diagram $\frac{T_{c}}{J}$ versus $\frac{E}{J}, \frac{H}{J}$ in the range of parameters where there is a single transition.

FIG. 6. Three dimensional phase diagram $\frac{T_{c}}{J}$ versus $\frac{E}{J}, \frac{H}{J}$ restricted to the range of parameters where the reentrant transition takes place. 


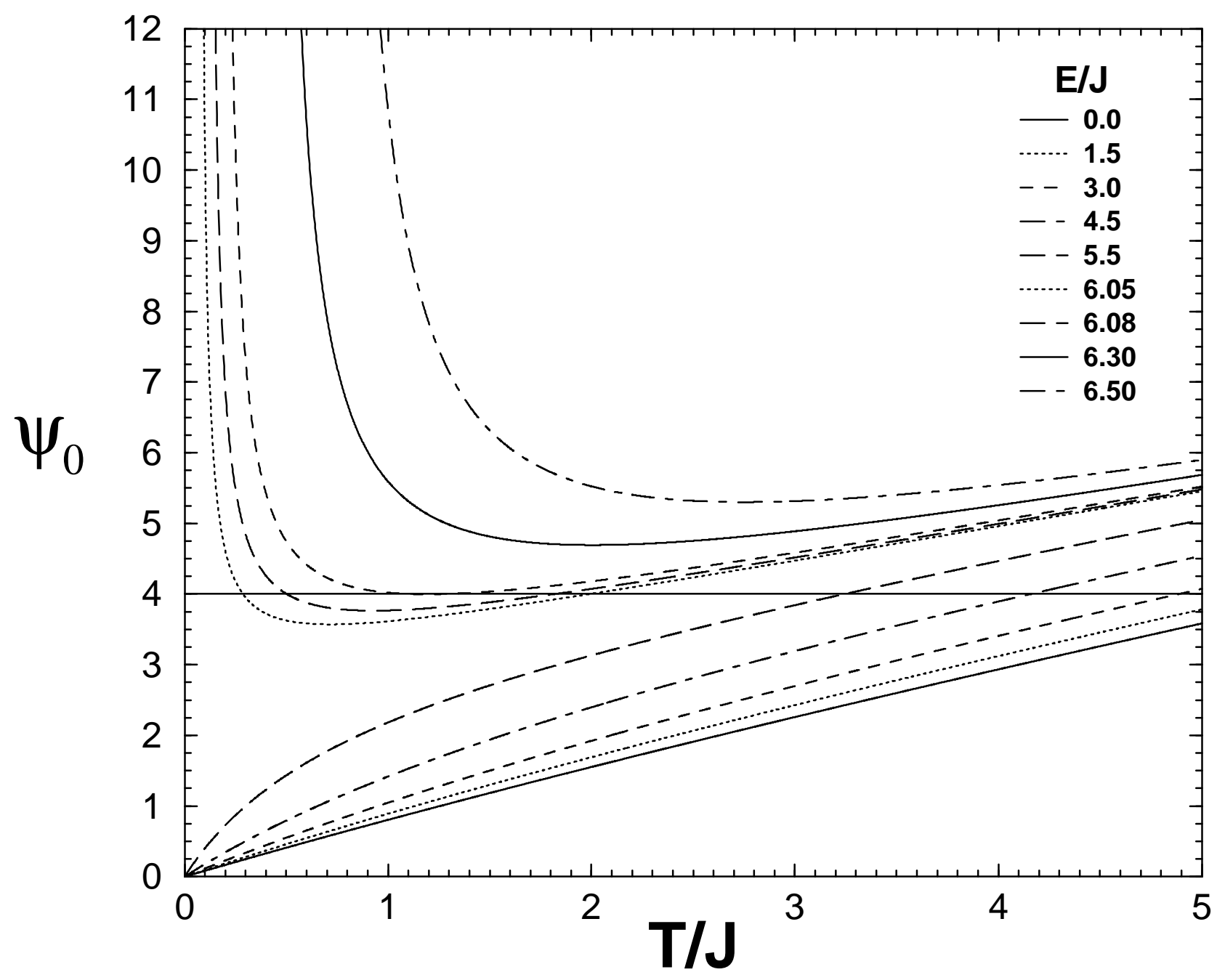

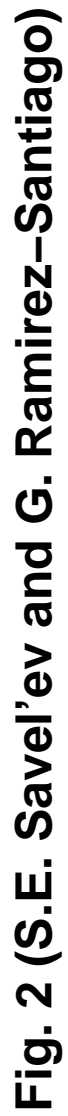



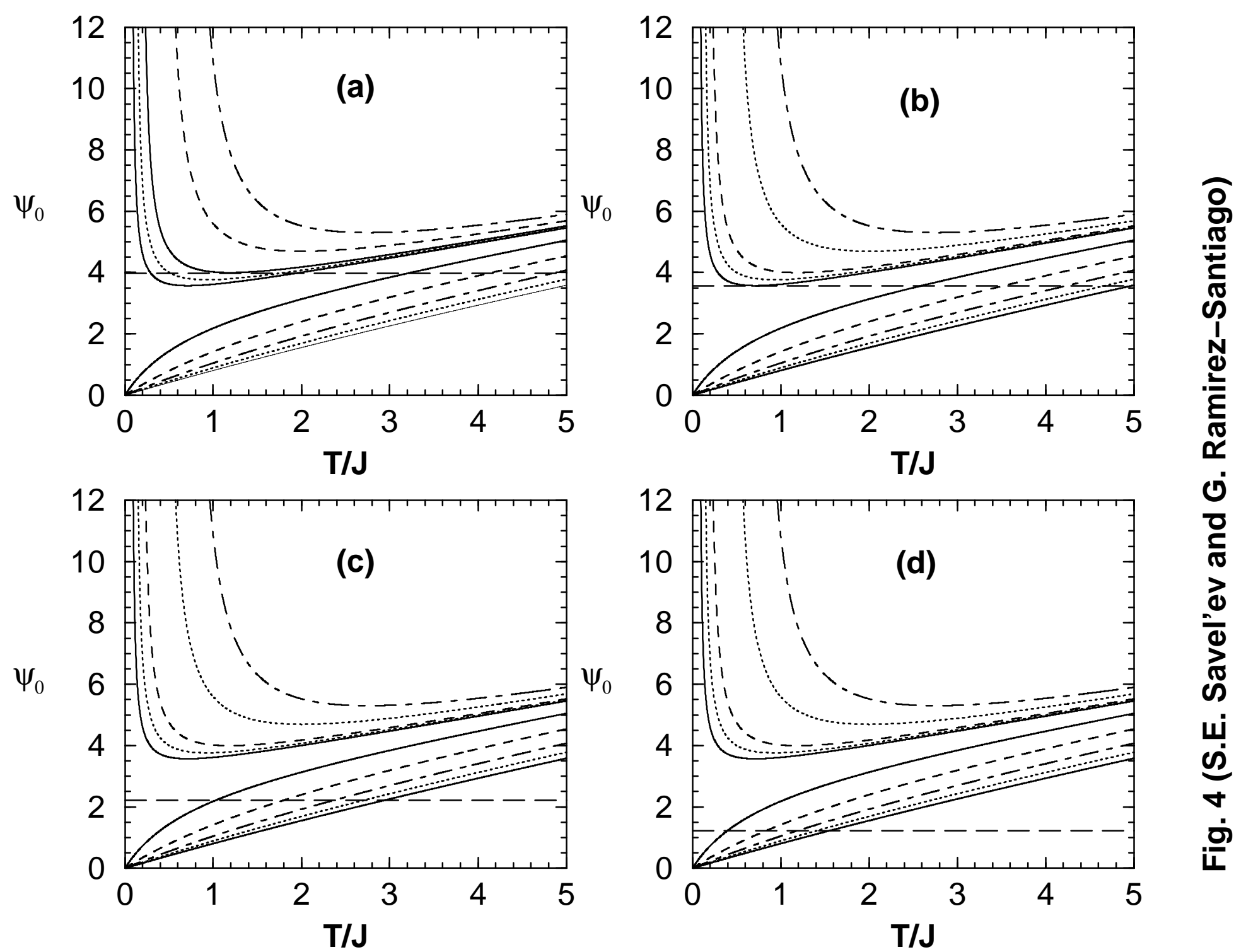


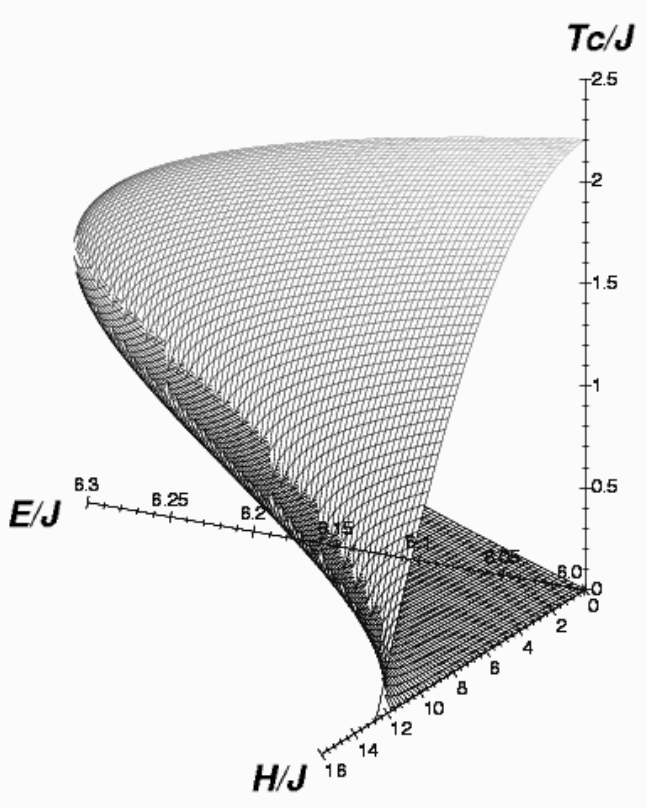

ig. 6 (S.E. Savel'ev and G. Ramirez-Santiago) 


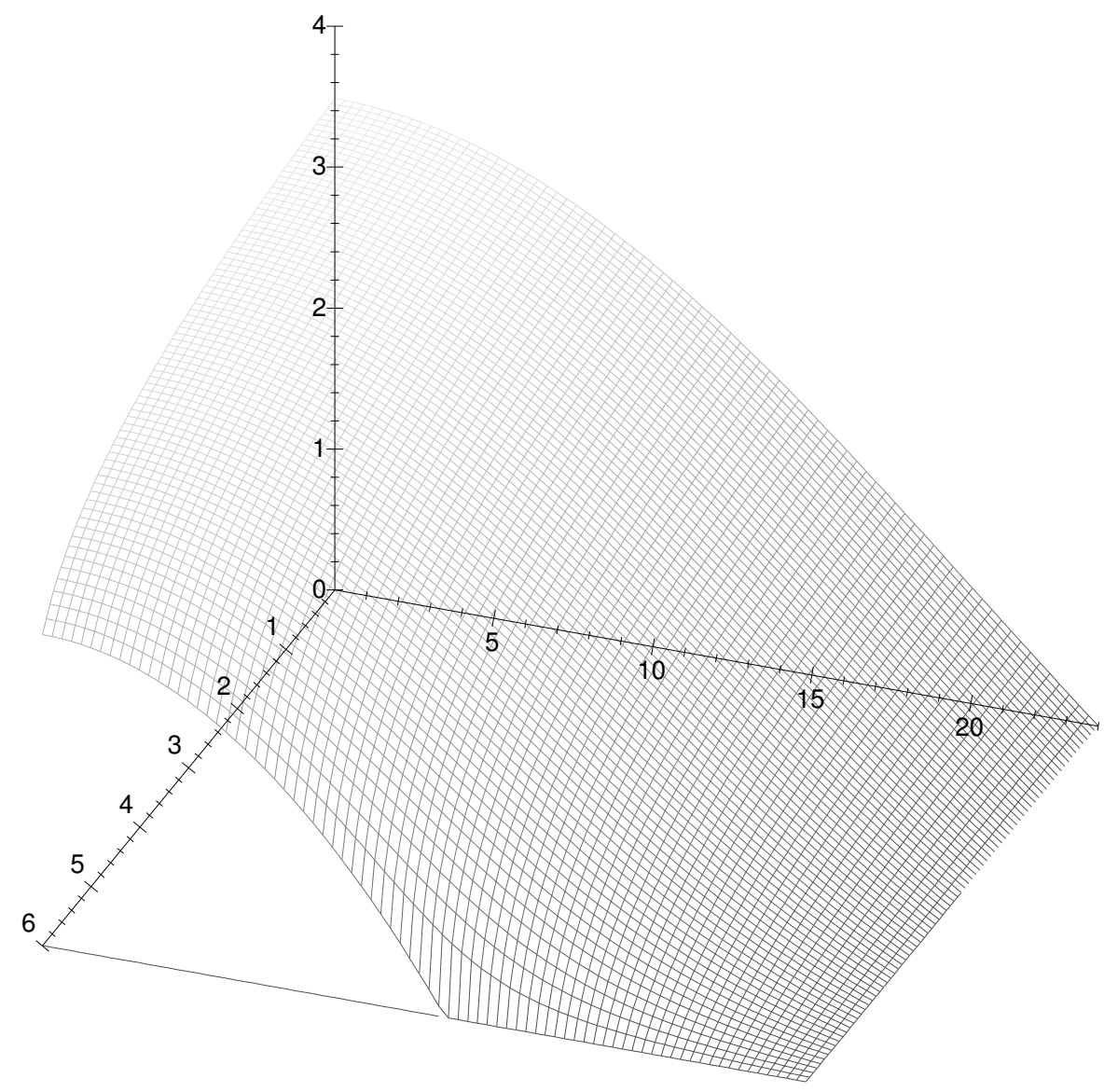

Fig. 5 (S.E. Savel'ev and G. Ramirez-Santiago) 


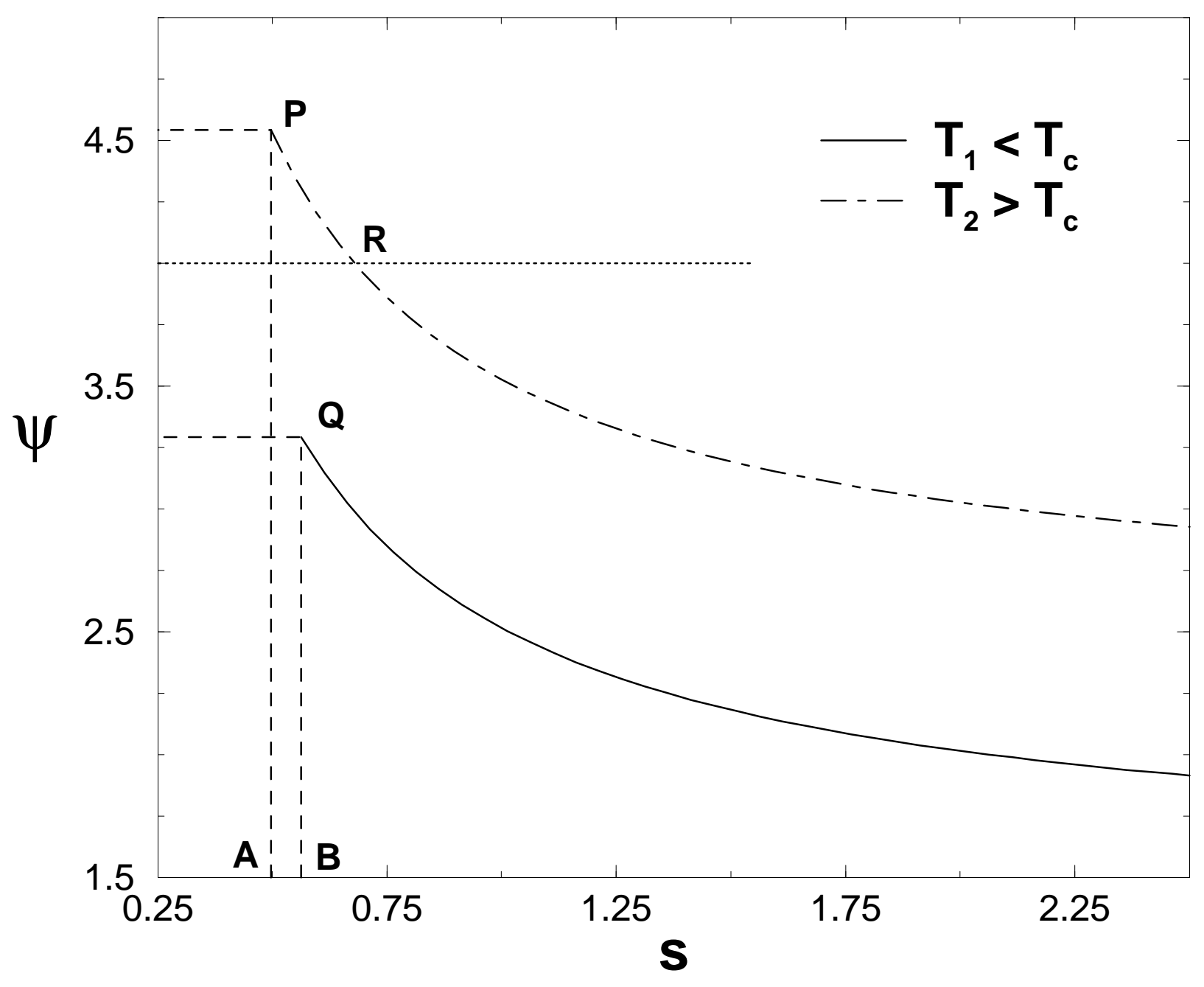

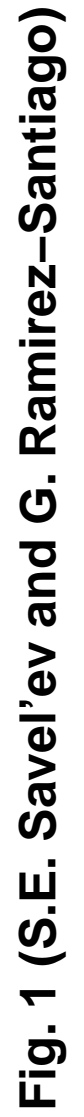




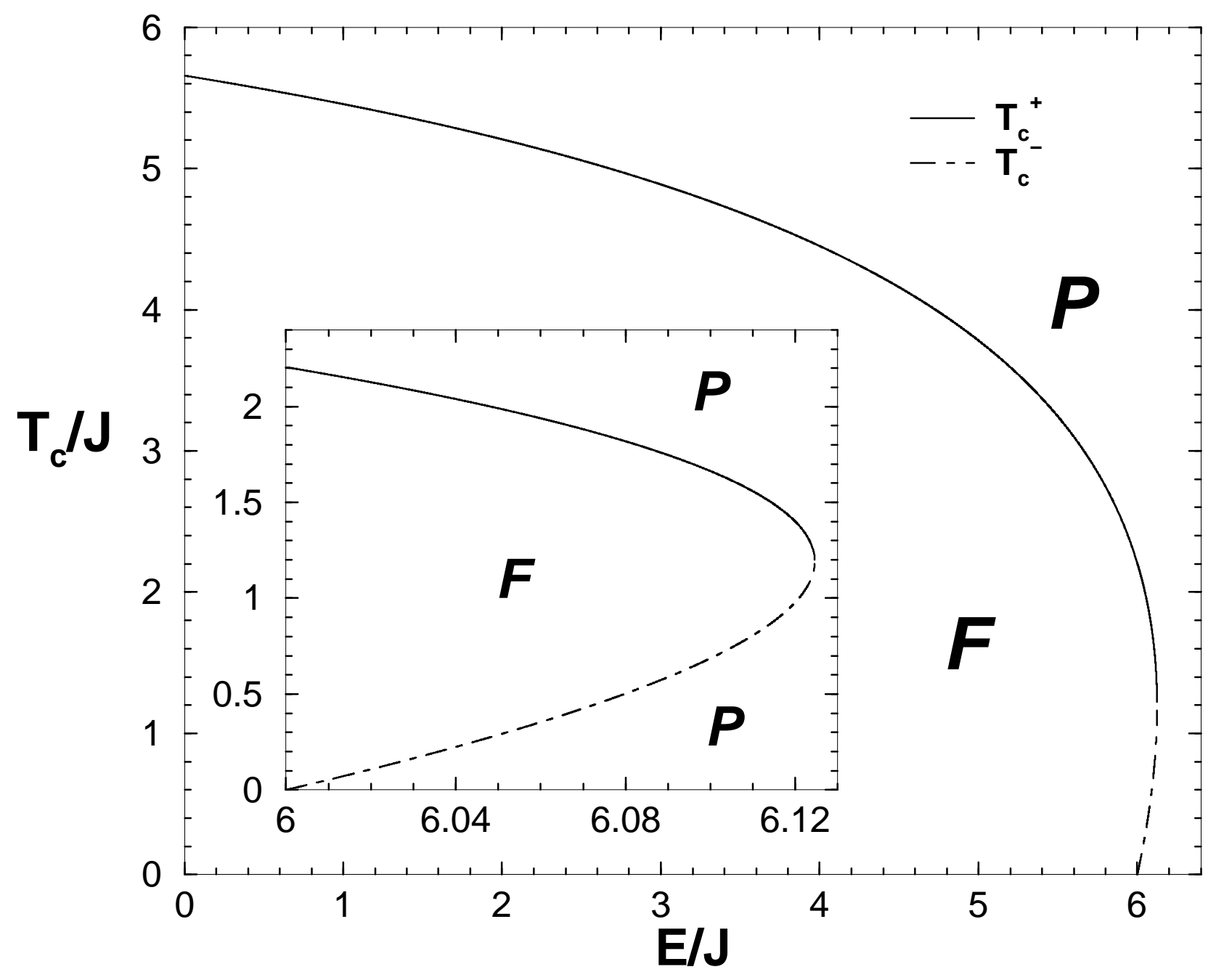

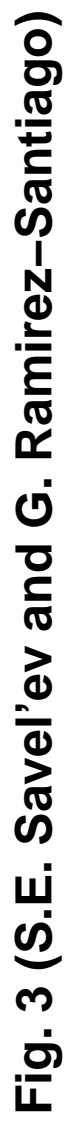

